\title{
Análise exploratória aplicada no estudo de medicamentos contendo piroxicam
}

\author{
Graciele Parisotto', Jorcem Simon de Souza', Marco Flôres Ferrão ${ }^{2,3,}{ }^{,}$, João Carlos Furtado ${ }^{3}$, \\ Rolf Fredi Molz ${ }^{3}$
}

${ }^{1}$ Curso de Farmácia, ${ }^{2}$ Departamento de Química e Física - Grupo de Quimiometria, ${ }^{3}$ Grupo de Pesquisa em Sistemas e Processos Industriais - Departamento de Informática, Universidade de Santa Cruz do Sul

*Correspondência:

M. F. Ferrão

UNISC, Av. Independência, 2293,

96815-900, Santa Cruz do Sul, RS,

Brasil.

E-mail: ferrao@unisc.br

\begin{abstract}
A identificação de diferentes formulações de medicamentos manipulados contendo piroxicam foi estudada, empregando espectros de reflexão difusa no infravermelho médio com transformada de Fourier (DRIFTS), em associação com a técnica de análise por agrupamentos hierárquicos (AAH). Os espectros de amostras, de 5 diferentes farmácias de manipulação, contendo piroxicam (10 mg e $20 \mathrm{mg}$ ) e seus respectivos excipientes, foram adquiridos em um espectrofotômetro NICOLET Magna 550, obtendo-se duas réplicas de cada amostra. Para a análise multivariada, as informações espectrais foram tratadas no programa Pirouette ${ }^{\circledR} 2.7$ da Infometrix, utilizando-se as regiões espectrais 1340 a $1470 \mathrm{~cm}^{-1}, 1535$ a $1680 \mathrm{~cm}^{-1}, 2800$ a $3004 \mathrm{~cm}^{-1} e$ 3290 a $3400 \mathrm{~cm}^{-1}$. Os dendogramas foram construidos com os dados auto-escalados, e correção do espalhamento da luz (MSC), utilizando três tipos de construção: simples, flexivel e incremental. Com a aplicação da análise hierárquica de agrupamentos constatou-se a formação de dois grupos distintos, um contendo os princípios ativos, e outro contendo os excipientes. Os resultados demonstram que a técnica DRIFTS em conjunto com análise por agrupamentos hierárquicos constitui uma alternativa para o controle de qualidade dos processos de produção de medicamentos.
\end{abstract}

Unitermos

- Antiinflamatórios

não-esteróides

- Infravermelho

- Piroxicam

- Análise multivariada

- Controle de qualidade de medicamentos

\section{INTRODUÇÃO}

Os medicamentos vêm sendo cada vez mais objetos de preocupação e de inúmeras pesquisas realizadas mundialmente. Estas pesquisas dizem respeito à prática médica, reações adversas, aumento e disseminação de resistência bacteriana à antibióticos, padrão e influência da propaganda na prescrição de medicamentos na área clínica, como também na área odontológica (Castilho, Paixão, Perini, 1999).

O uso do medicamento como forma de competição pelo setor farmacêutico tem se tornado abusivo, mas o avanço do medicamento genérico mantém o mercado mais equilibrado e com o uso mais racional dos medica- 
mentos fornecendo, assim, ao consumidor um medicamento de mesma qualidade e menor custo (Castilho, Paixão, Perini, 1999).

Por tais razões, é de enorme importância o desenvolvimento de métodos eficazes e rápidos para maior controle de qualidade dos medicamentos comercializados, tornando-se imprescindível o desenvolvimento de rotinas de análises que, além de servirem para este propósito, favoreçam sua implantação em comparação aos métodos usuais.

Neste sentido, a espectroscopia no infravermelho vem sendo amplamente estudada, como por exemplo, a técnica de reflexão no infravermelho próximo (NIRR) que abrange a faixa entre 1300 a $2500 \mathrm{~nm}$, que vem sendo utilizada em análises farmacêuticas (Blanco et al., 1999a; Herkert, Prinz, Kovar, 2001; Pasquini, 2003). Este desenvolvimento da técnica se deve à adequada determinação qualitativa e quantitativa de parâmetros físicos e químicos, habilidade no processo, além de ser compatível com a fibra ótica, fator este que acaba por facilitar as análises. Um exemplo desta aplicação é apresentado por Blanco et al. (1999b), envolvendo miocamicina, um bacteriostático, como princípio ativo. Neste trabalho, o método NIRR utilizado foi validado como alternativa para um método espectrofotométrico de referência.

Por outro lado, o emprego de técnicas de reflexão difusa no infravermelho médio (DRIFTS - Espectroscopia por reflexão difusa no infravermelho médio com transformada de Fourier) que abrangem a faixa espectral de 400 a $4000 \mathrm{~cm}^{-1}$, vem sendo cada vez mais difundido (Yang e Irudayaraj, 2002). Uma experimentação recente da técnica foi apresentada no trabalho de Sabin et al. (2003), em que foi aplicado na identificação de diferentes concentrações de fármacos antidepressivos, obtendo resultado satisfatório.

\section{Antiinflamatório Não-Esteróide}

O piroxicam é um antiinflamatório não-esteróide, que atua inibindo a atividade da enzima ciclooxigenase, que catalisa a biossíntese das prostaglandinas e tromboxanos a partir do ácido araquidônico. O processo inflamatório consiste na resposta orgânica mais precoce diante de lesão tissular ou infecção, sendo que este processo fisiológico envolve uma ação coordenada entre o sistema imunológico e o tecido no qual ocorreu a lesão. Piroxicam é utilizado no tratamento de artrite gotosa aguda, artrite reumatóide, inflamação não-reumática e osteartrite (Korolkovas, 2002).

O piroxicam corresponde quimicamente ao 4hidróxi-2-metil-N-(piridinil-2)-2H-1,2-benzotiazina-3carboxamida-1,1-dióxido (Merck, 2001). Trata-se de um pó branco a levemente amarelo, cristalino, praticamente insolúvel em água, solúvel em cloridrato de metileno, levemente solúvel em etanol (European, 2001). Possui peso molecular de 331,35g/mol, com fórmula estrutural apresentada na Figura 1.

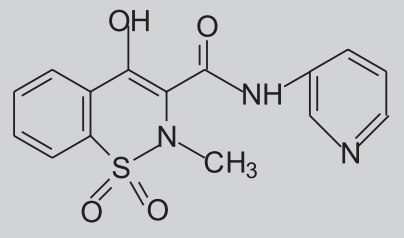

FIGURA 1 - Fórmula estrutural do piroxicam.

Seu ponto de fusão varia de 198 a $200^{\circ} \mathrm{C}$. Para sua identificação pode ser empregada a espectroscopia no infravermelho, comparando a um piroxicam de referência, a espectrofotometria no ultravioleta, ou, ainda, a cromatografia líquida e a cromatografia em camada delgada (European, 2001; USP 25, 2002).

Quando empregamos o infravermelho são preparados discos de piroxicam em $\mathrm{KBr}$. Se o espectro obtido em um estado sólido mostrar diferenças, dissolve-se a substância a ser examinada e a substância de referência, separadamente, em um volume mínimo de cloreto de metileno, evaporando até a secura num banho-maria. Finalmente registra-se um novo espectro usando os resíduos, comparando-os (European Pharmacopéia, 2001).

Como nova alternativa para a identificação de formulações contendo piroxicam é proposta a utilização da análise multivariada associada a métodos espectroscópicos por reflexão difusa no infravermelho com transformada de Fourier (DRIFTS). Estes métodos possibilitam a verificação da similaridade de diversos fármacos nas diferentes etapas de produção (Blanco, 2000).

Os espectros de reflexão difusa em conjunto com a análise multivariada envolvem grande número de variáveis, permitindo construir uma estrutura de grupos AAH (Análise por Agrupamentos Hierárquicos). Segundo Lavine (2002), a Análise por Agrupamentos Hierárquicos consiste em descobrir grupos de amostras dentro do conjunto de dados, onde cada amostra é tratada como um ponto num espaço n-dimensional, avaliando a similaridade entre as mesmas. Mede-se a distância entre seus pontos no espaço agrupando-as com base nas suas semelhanças e diferenças associadas a mais de uma freqüência ao mesmo tempo. Como vantagens adicionais são necessárias pequenas quantidades de amostra, não sendo preciso a separação dos componen- 
tes da formulação e ainda não ocorre geração de resíduos nocivos ao ambiente.

Desta forma, o presente trabalho tem como objetivo aplicar a análise multivariada em dados de espectroscopia no infravermelho para identificar a similaridade entre medicamentos contendo piroxicam, bem como de seus excipientes mais comuns empregados em farmácia de manipulação.

\section{ANÁLISE MULTIVARIADA}

Os avanços na microinformática e instrumentação no final do último século, juntamente com as ferramentas de Análise Multivariada (AM) tornaram possível manipular dados de absorbância espectral associados a mais de uma freqüência ao mesmo tempo. Estes métodos têm possibilitado modelar propriedades químicas e físicas de sistemas simples ou complexos a partir de seus dados espectroscópicos, como no caso do estudo de formulações farmacêuticas (Gabrielsson, 2002; Pasquini, 2003; Yoon et al., 2004). Por outro lado, as ferramentas de Análise Multivariada permitem modelar sistemas onde estão sobrepostas informações espectrais ou cromatográficas de vários componentes através de várias regiões espectrais selecionadas para análise (Sabin, Banet, Ferrão, 2003; Sabin, Ferrão, Furtado, 2004). Os dados são representados por uma matriz, onde as linhas representam as amostras e as colunas as variáveis (comprimentos de onda) (Lavine, 2002; Sabin, Banet, Ferrão, 2003). Pode-se empregar métodos de compressão da dimensionalidade como a Análise por Componentes Principais (ACP), ou simplesmente calcular a distância n-dimensional entre cada linha da matriz (amostra), empregando-se conceito de similaridade (Ferreira et al., 2002; Gabrielsson, Lindberg, Lundstedt, 2002; Silva et al., 2002).

\section{ANÁLISE POR AGRUPAMENTOS HIERÁRQUICOS (AAH)}

Este método tem por finalidade verificar a semelhança entre as linhas (amostras) de uma matriz de dados (espectros), calculando-se a distância no espaço ndimensional (variáveis), na maioria das vezes, a distância Euclidiana, conforme expressão 1.

$$
d_{a b}=\sqrt{\sum_{i=1}^{m}\left(x_{a i}-x_{b i}\right)^{2}}
$$

O resultado é apresentado na forma gráfica denominado dendograma (Ferreira, 2002; Lavine, 2002; Sabin, Banet, Ferrão, 2003) assemelhando-se a uma estrutura em forma de árvore, sendo que $\mathrm{d}_{\mathrm{ab}}$ corresponde à distância entre os pontos a e $b, \mathrm{e}_{\text {máx }}$ representa a distância entre qualquer par de pontos (amostras), onde são informadas a similaridade conforme expressão 2 .

$S_{a b}=1-\frac{d_{a b}}{d_{\text {max }}}$

Utilizam-se vários métodos para calcular a similaridade entre as amostras. No presente estudo foram empregados os métodos de construção simples, incremental e flexível definidos pelas expressões 3, 4 e 5, respectivamente (Infometrix, 1999).

$$
\begin{aligned}
& d_{a b \Rightarrow c}=0,5 d_{a c}+0,5 d_{b c}-0,5\left|d_{a c}-d_{b c}\right| \\
& d_{a b \Rightarrow c}=\frac{\left(\left(n_{a}+n_{c}\right) d_{a c}^{2}+\left(n_{b}+n_{c}\right) d_{b c}^{2}-n_{c} d_{a b}^{2}\right)^{1 / 2}}{n_{a}+n_{b}+n_{c}} \\
& d_{a b \Rightarrow c}=\left(0,625 d_{a c}^{2}+0,625 d_{b c}^{2}+0,25 d_{a b}^{2}\right)^{1 / 2}
\end{aligned}
$$

onde $n_{i}$ corresponde ao número de amostras no grupo $i$.

\section{MÉTODOS E MATERIAIS}

\section{Amostragem}

Foram empregadas amostras de piroxicam (10 e $20 \mathrm{mg}$ ) de cinco diferentes farmácias de manipulação e seus respectivos excipientes. As diferentes concentrações das amostras foram identificadas por A e B, sendo A para o piroxicam $10 \mathrm{mg}$ e B para o piroxicam $20 \mathrm{mg}$. Os cinco fornecedores foram diferenciados por 1, 2, 3, 4 e 5 . Os excipientes contendo somente amido foram identificados pela letra $\mathrm{C}$ e o excipiente composto por D. Este último, quando pertencente ao fornecedor 1 contém, além de amido, talco, estearato de magnésio e lauril sulfato de sódio (D1), e quando pertencente ao fornecedor 4 era composto por amido, celulose, estearato de magnésio e lauril sulfato de sódio (D4). As duplicatas foram identificadas por a e b.

\section{Aquisição dos espectros}

Espectros em duplicata para cada uma das concentrações do princípio ativo (piroxicam 10 e $20 \mathrm{mg}$ ) e para cada um dos seus excipientes foram coletados num espectrofotômetro NICOLET Magna 550, com resolução de $4 \mathrm{~cm}^{-1}$ e 32 varreduras. $\mathrm{O}$ instrumento foi equipado com um acessório de reflectância difusa para aquisição dos espectros. 


\section{Análise multivariada}

Para a análise hierárquica de agrupamentos (AHA) as informações espectrais foram tratadas no aplicativo Pirouette ${ }^{\circledR}$ da Infometrix. As regiões onde se evidenciou pouca informação espectral, bem como aquelas onde o ruído no espectro devido ao vapor d'água e o dióxido de carbono estavam presentes, foram excluídas. Portanto, os modelos desenvolvidos foram realizados sobre as regiões espectrais compreendidas entre: 1340 a $1470 \mathrm{~cm}^{-1}, 1535$ a $1680 \mathrm{~cm}^{-1}, 2800$ a $3004 \mathrm{~cm}^{-1}$ e 3290 a $3400 \mathrm{~cm}^{-1}$, conforme Figura 2.

Posteriormente foram experimentadas várias formas para a construção dos dendogramas como simples, flexí-

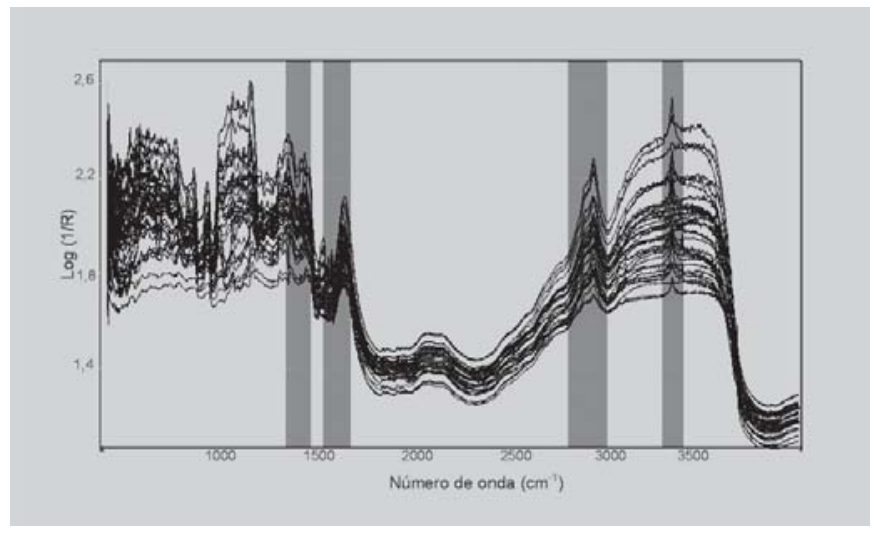

FIGURA 2 - Regiões espectrais empregadas na análise multivariada dos dados DRIFTS. vel e incremental, bem como pré-processamentos (os dados auto-escalados ou centrados na média) e ainda, foi empregada à matriz de dados a técnica da correção do espalhamento da luz (MSC) (Olinger, Griffiths, 1993; Pakisatan et al., 2001), devido a possíveis diferenças na granulometria entre princípio ativo e excipiente. Também foram experimentadas outras transformações nos espectros em substituição ao MSC como normalização e primeira derivada, porém estes tratamentos sempre apresentaram resultados inferiores àqueles nos quais empregouse o MSC.

\section{RESULTADOS E DISCUSSÃO}

Os espectros de piroxicam 10 e $20 \mathrm{mg}$ e seus respectivos excipientes, obtidos a partir de DRIFTS, foram modelados empregando-se os dados auto-escalados com construções simples, flexível e incremental com tratamento MSC, os quais apresentaram os melhores resultados, sendo mostrados pelas Figuras 3 e 4. Os tipos de construção flexível e incremental apresentaram resultado de similaridade equivalentes.

O dendograma no qual se empregou a conexão simples (Figura 3 ) agrupou a grande maioria das duplicatas dos espectros, mas não houve boa discriminação, tanto dos princípios ativos quanto dos seus respectivos excipientes.

Já na Figura 4, o dendograma apresentado com a construção incremental (equivalente ao da flexível), mos-

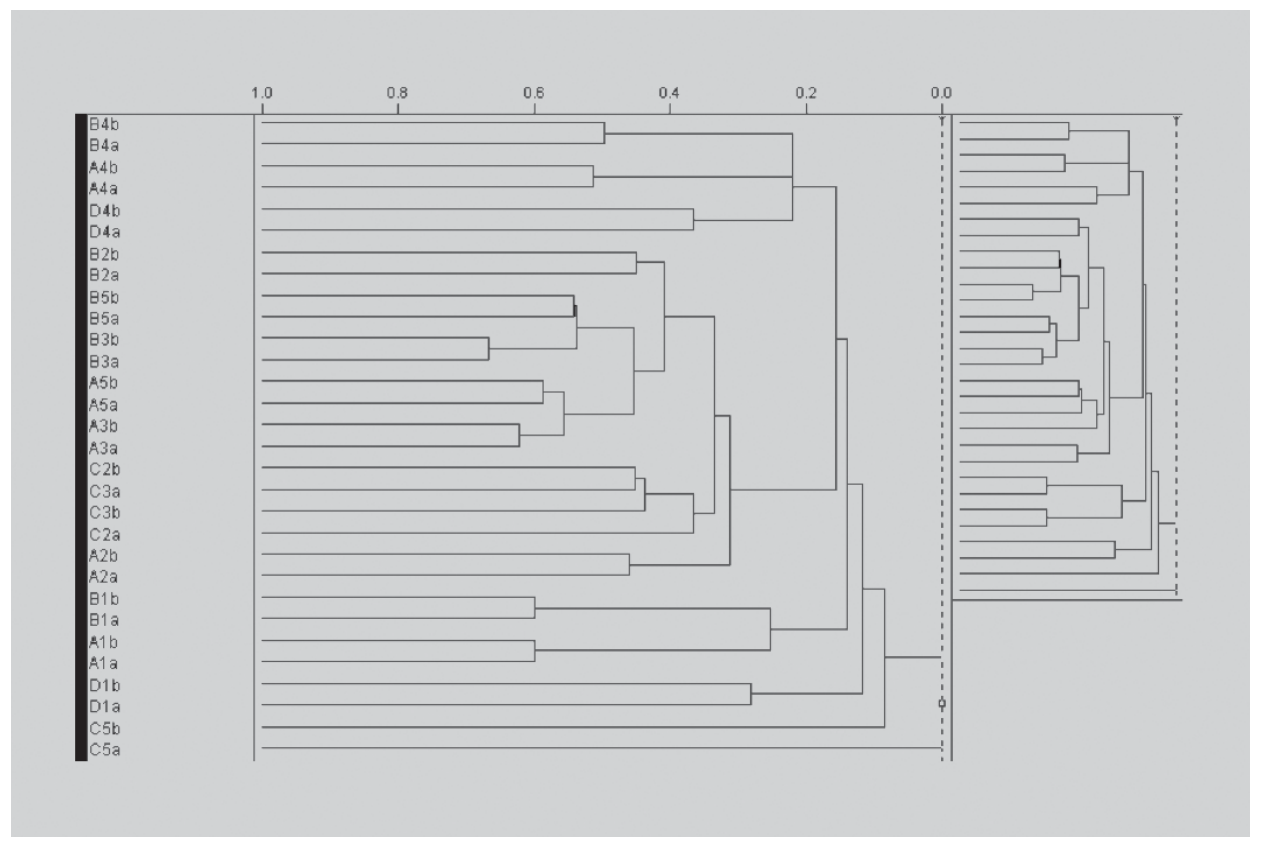

FIGURA 3 - Dendograma obtido a partir de espectros DRIFTS de amostras contendo piroxicam 10 e $20 \mathrm{mg}$, e seus excipientes, empregando os dados auto-escalados, utilizando-se MSC e construção simples. 


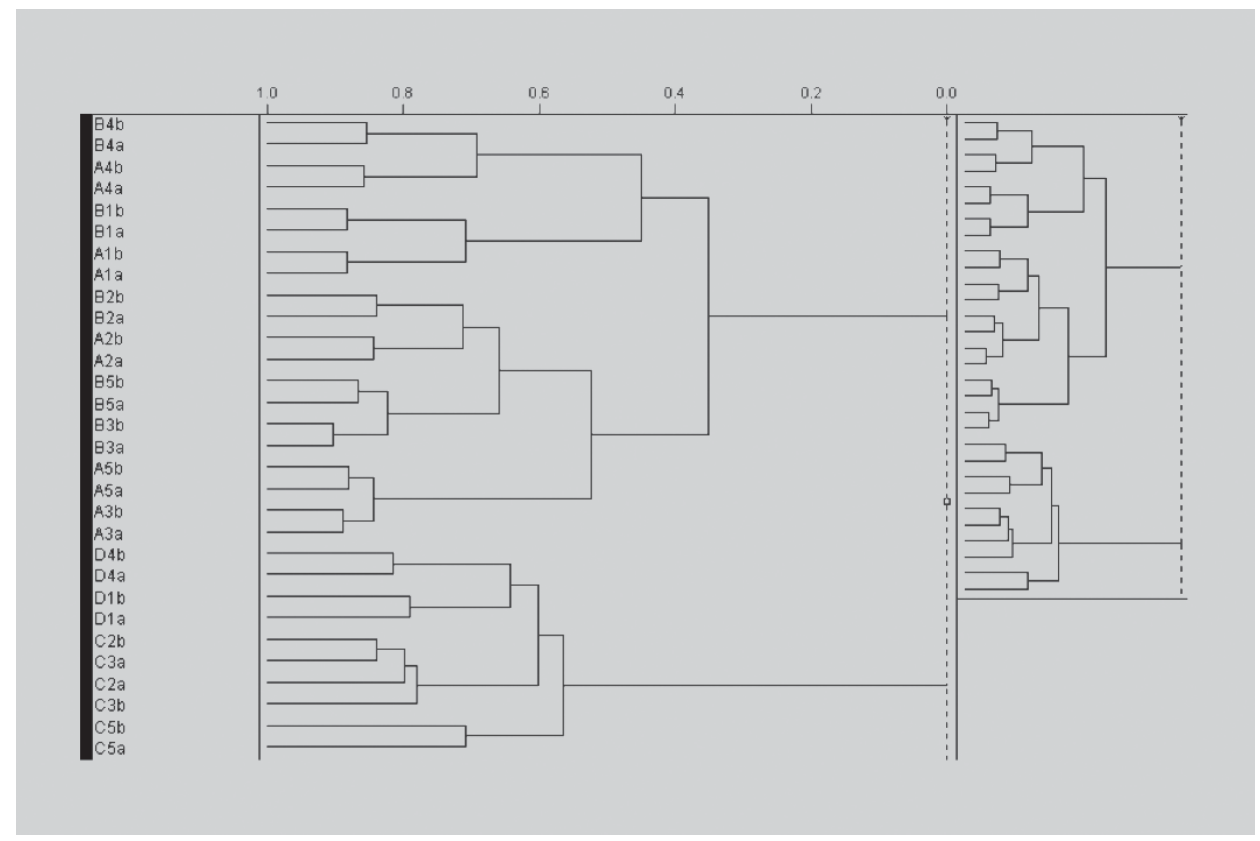

FIGURA 4 - Dendograma obtido a partir de espectros DRIFTS de amostras contendo piroxicam 10 e 20mg, e seus excipientes, empregando os dados auto-escalados, utilizando-se MSC e construção incremental.

tra boa discriminação, tanto dos princípios ativos quanto de seus excipientes, que além de aumentar a similaridade das duplicatas para cada amostra, as agrupa segundo suas características químicas (semelhança espectral).

Ao se observar o dendograma da Figura 4, verificouse a formação de 2 grupos distintos, um contendo os princípios ativos, amostras de piroxicam 10 (A) e $20 \mathrm{mg}$ (B) e outro contendo os respectivos excipientes. No grupo das amostras contendo princípios ativos, as amostras 1, 2 e 4 foram agrupadas por fornecedor e as amostras 3 e 5 formaram subgrupos segundo as diferentes dosagens.

Dentro do grupo dos excipientes, os formados somente por amido (C) são discriminados em relação ao excipiente composto (D), devido as amostras deste último grupo conterem, além do amido, lauril sulfato de sódio, talco, estearato de magnésio e aerosil (D1) e para as réplicas D4 apenas o talco é substituído por celulose, constituintes que também apresentaram absorção significativa na região espectral estudada. Dentre os excipientes formados por amido, todos possuem um pouco de aerosil, visto que os fornecedores 2 e 3 apresentaram mais similaridade, em virtude de apresentarem quantidades próximas de aerosil.

Também, pode-se considerar que a presença do excipiente composto nos fornecedores 1 e 4 foi responsável pela subdivisão do piroxicam $10 \mathrm{mg}$ (A) e $20 \mathrm{mg}$ (B) dos demais fornecedores, devido a presença das diferentes matérias-primas (excipiente D).

\section{CONCLUSÕES}

A técnica DRIFTS em conjunto com a análise multivariada permitiu construir uma estrutura de grupos a partir da aplicação da análise por agrupamentos hierárqui$\cos (\mathrm{AAH})$, na qual constatou-se a formação de dois grupos distintos, um contendo os princípios ativos e outro contendo os excipientes.

A técnica possui como vantagem o envolvimento de grande número de variáveis, permitindo construir uma estrutura de grupos, com suas semelhanças e diferenças associadas a mais de uma freqüência ao mesmo tempo, não havendo necessidade de separação dos componentes da formulação. Também sendo uma análise não destrutiva, não produz geração de resíduos nocivos ao meio ambiente, além de necessitar pequena quantidade de amostra para a análise.

Estes resultados demonstram que a técnica DRIFTS em conjunto com o método AAH constitui uma alternativa para verificação da presença de fármacos em medicamentos manipulados, além de permitir inferir sobre a semelhança/diferenças entre medicamentos de distintas farmácias de manipulação.

\section{AGRADECIMENTOS}

Os autores agradecem as bolsas de iniciação científica concedidas pela Fundação de Amparo a Pesquisa do 
Estado do Rio Grande do Sul e pelo apoio do Curso de Química e da Central Analítica da UNISC.

\section{ABSTRACT}

\section{Exploratory analysis applied in study of pharmaceutical formulations with piroxicam}

The identification of different pharmaceutical formulations with piroxicam was studied, using spectra of diffuse reflectance infrared Fourier transform spectroscopy (DRIFTS), in association with hierarchical cluster analysis (HCA) technique. The spectra of samples of the 5 different compounding pharmacies, containing 10 or $20 \mathrm{mg}$ of piroxicam and its respective inactive ingredients, had been collected in Nicolet Magna 550 spectrophotometer. For the multivariate analysis, the spectral information had been processed in software package Pirouette ${ }^{\circledR} 2.7$ of the Infometrix. The dendograms had been constructed with the autoscaled data, and multiplicative scatter correction (MSC), using three types of linkage methods: single, flexible and incremental. By applying the hierarchical cluster analysis the formation of two distinct groups: one with the active principles and another group with the inactive ingredients was proved. These results demonstrate that the DRIFTS associated with chemometrics tools, constitute a quality control alternative of drugs production processes.

UNITERMS: Non-steroidal anti-inflammatory drugs (NSAIDs). DRIFTS. Piroxicam. Multivariate analysis. Drugs quality control.

\section{REFERÊNCIAS BIBLIOGRÁFICAS}

BLANCO M., COELlO J., EUSTAQUiO, A., ITURRIAGA H., MASPOCH S. Analytical control of pharmaceutical production steps by near infrared reflectance spectroscopy. Anal. Chim. Acta, Amsterdam, v. 392, n. 2-3, p.237-246, 1999a.

BLANCO M., COELLO J., EUSTAQUIO A., ITTURRIAGA H., MASPOCH S. Development and validation of methods for the determination of miokamycin in various pharmaceutical preparations by use of near infrared reflectance spectroscopy. Analyst, London, v. 124, n. 7, p. 1089-1092, 1999 b.

BLANCO, M.; COELHO, J.; ITURRIAGA, H.; MASPOCH, S.; POU, N. Development and validation of a near infrared method for the analytical control of a pharmaceutical preparation in three steps of the manufacturing process. Fresenius J. Anal. Chem., Berlin, v. 368, n.5, p. $534-539,2000$.

CASTILHO, L.S; PAIXÃO, H.H; PERINI, E. Prescrição de medicamentos de uso sistêmico por cirurgiões-dentistas, clínicos gerais. Rev. Saúde Pública., São Paulo, v. 33, n.3, p. 287-294, 1999.

EUROPEAN Pharmacopoeia 2002. 4 Ed. Strasbourg: Council of Europe, 2001. p. 1771.

FERREIRA, E.C.; RODRIGUES, S.H.B.G.; FERREIRA, M.M.C.; NÓBREGA, J.A.; NOGUEIRA, A.R.A. Análise exploratória dos teores de constituintes inorgânicos em sucos e refrigerantes de uva. Eclet. Quím., São Paulo, v. 27, n.spe, p. 77-90, 2002.

FERREIRA, M.M.C. Multivariate QSAR. J. Braz. Chem. Soc., São Paulo, v.13, n.6, p.742-753, 2002.

GABRIELSSON, J.; LINDBERG, N.-O.; LUNDSTEDT, T. Multivariate methods in pharmaceutical applications. $J$. Chemom., Chichester, v. 16, n.3, p. 141-160, 2002.

HERKERT T., PRINZ H., KOVAR K. One hundred percent online identity check of pharmaceutical products by nearinfrared spectroscopy on the packaging line. Eur. J. Pharm. Biopharm., London, v. 51, n.1, p.9-16, 2001.

INFOMETRIX, Inc., Pirouette 2.70, Woodinville, WA, 1999.

KOROLKOVAS, A. Dicionário terapêutico Guanabara 2002/2003. Rio de Janeiro: Guanabara Koogan, 2002. p. 21.1-21.10.

LAVINE, B.K. Clustering and classification of analytical data. In: MEYERS, R.A. (Ed.) Encyclopedia of Analytical Chemistry. Chichester: John Wiley \& Sons, Ltd, 2002. v. 16, p. 9689-9710.

OLINGER, J.M.E.; GRIFFITHS, P.R. Effects of sample dilution and particle size/morphology on diffuse reflection spectra of carbohydrate systems in the nearand mid- infrared. Part II: Durum wheat. Appl. Spectrosc., Baltimore, v. 47, p. 695-701, 1993.

PASIKATAN, M.C.; STEELE, J.L.; SPILLMAND, C.K.; HAQUE, E. Near infrared reflectance spectroscopy for online particle size analysis of powders and ground materials. J. Near Infrared Spectrosc., Sussex, v. 9, n.3, p. 153-164, 2001. 
PASQUINI, C. Near infrared spectroscopy: fundamentals practical aspects and analytical applications. J. Braz. Chem. Soc., São Paulo, v. 14, n.2, p. 198-219, 2003.

SABIN, J.G.; BANET, E.V; FERRÃO, M.F. Análise exploratória aplicada na identificação de fármacos antidepressivos. Parte I: Análise Hierárquica de Agrupamentos. Tecno-lóg., Santa Cruz do Sul, v. 7, n.1, p. 69-88, 2003.

SABIN, J.G.; FERRÃO, M.F; FURTADO, J.C. Análise multivariada aplicada na identificação de fármacos antidepressivos. Parte II: Análise por componentes principais (PCA) e o método de classificação SIMCA. Rev. Bras. Ciênc. Farm., São Paulo, v. 40, n.3, p. 387396, 2004.

SILVA, F.V.; KAMOGAWA, M.Y.; FERREIRA, M.M.C.; NÓBREGA, J.A.; NOGUEIRA, A.R.A. Discriminação geográfica de águas minerais do Estado de São Paulo através da análise exploratória. Eclet. Quím., São Paulo, v. 27, n.spe, p. 91-102, 2002.
THE MERCK INDEX: an encyclopedia of chemicals, drugs, and biologicals. Thirteenth edition. New Jersey: Merck, 2001. p.7588.

UNITED States Pharmacopoeia. 25.ed. Rockille: United States Pharmacopeial Convention, 2002. p.1388-1389.

YANG H., IRUDAYARAJ J. Rapid determination of vitamin C by NIR, MIR and FT-Raman techniques. J. Pharm. Pharmacol., London, v.54, n.9, p.1247-1255, 2002.

YOON W.L., JEE R.D., CHARVILLA., LEE G., MOFFAT A.C. Application of near-infrared spectroscopy to the determination of the sites of manufacture of proprietary products. J. Pharm. Biomed. Anal., Amsterdam, v.34, n.5, p.933-944, 2004.

Recebido para publicação em 03 de março de 2005. Aceito para publicação em 11 de novembro de 2005. 